\title{
KMT-2021-BLG-0912Lb: A microlensing super Earth around a K-type star
}

\author{
Cheongho $\mathrm{Han}^{01}$, Ian A. Bond ${ }^{02}$, Jennifer C. Yee ${ }^{03}$, Weicheng Zang ${ }^{04}$ \\ (Leading authors) \\ Michael D. Albrow ${ }^{05}$, Sun-Ju Chung ${ }^{06}$, Andrew P. Gould ${ }^{07,08}$, Kyu-Ha Hwang ${ }^{06}$, Youn Kil Jung ${ }^{06}$, Doeon Kim ${ }^{01}$, \\ Chung-Uk Lee ${ }^{06}$, Yoon-Hyun Ryu ${ }^{06}$, In-Gu Shin ${ }^{06}$, Yossi Shvartzvald ${ }^{09}$, Sang-Mok Cha ${ }^{06,10}$, Dong-Jin Kim ${ }^{06}$, \\ Seung-Lee Kim ${ }^{06,11}$, Dong-Joo Lee ${ }^{06}$, Yongseok Lee ${ }^{06}$, Byeong-Gon Park ${ }^{06,11}$, Richard W. Pogge ${ }^{08}$ \\ (The KMTNet Collaboration), \\ Fumio Abe ${ }^{12}$, Richard Barry ${ }^{13}$, David P. Bennett ${ }^{13,14}$, Aparna Bhattacharya ${ }^{13,14}$, Yuki Hirao ${ }^{15}$, Hirosane Fujii ${ }^{15}$, \\ Akihiko Fukui ${ }^{16,17}$, Yoshitaka Itow ${ }^{12}$, Rintaro Kirikawa ${ }^{15}$, Iona Kondo ${ }^{15}$, Naoki Koshimoto ${ }^{18}$, ${ }^{19}$, Yutaka Matsubara ${ }^{12}$, \\ Sho Matsumoto ${ }^{15}$, Yasushi Muraki ${ }^{12}$, Shota Miyazaki ${ }^{15}$, Clément Ran ${ }^{13}$, Arisa Okamura ${ }^{15}$, Nicholas J. Rattenbury ${ }^{20}$, \\ Yuki Satoh ${ }^{15}$, Takahiro Sumi ${ }^{15}$, Daisuke Suzuki ${ }^{21}$, Stela Ishitani Silva ${ }^{13,22}$, Taiga Toda ${ }^{15}$, Paul J. Tristram ${ }^{23}$, \\ Hibiki Yama ${ }^{15}$, Atsunori Yonehara ${ }^{15}$ \\ (The MOA Collaboration), \\ Tony Cooper ${ }^{24}$, Plamen Dimitrov ${ }^{24}$, Subo Dong ${ }^{25}$, John Drummond ${ }^{26,27}$, Jonathan Green ${ }^{24}$, Steve Hennerley ${ }^{24}$, \\ Zhuokai Liu ${ }^{25,28}$, Shude $\mathrm{Mao}^{04,29}$, Dan $\mathrm{MaOz}^{30}$, Matthew Penny ${ }^{31}$, and Hongjing Yang ${ }^{04}$ \\ (LCOGT \& $\mu$ FUN Follow-up Team),
}

(Affiliations can be found after the references)

Received ; accepted

\begin{abstract}
Aims. The light curve of the microlensing event KMT-2021-BLG-0912 exhibits a very short anomaly relative to a single-lens single-source form. We investigate the light curve for the purpose of identifying the origin of the anomaly.

Methods. We model the light curve under various interpretations. From this, we find four solutions, in which three solutions are found under the assumption that the lens is composed of two masses ( $2 \mathrm{~L} 1 \mathrm{~S}$ models), and the other solution is found under the assumption that the source is comprised of a binary-star system (1L2S model). The 1L2S model is ruled out based on the contradiction that the faint source companion is bigger than its primary, and one of the 2L1S solutions is excluded from the combination of the relatively worse fit, blending constraint, and lower overall probability, leaving two surviving solutions with the planet/host mass ratios of $q \sim 2.8 \times 10^{-5}$ and $\sim 1.1 \times 10^{-5}$. A subtle central deviation supports the possibility of a tertiary lens component, either a binary companion to the host with a very large or small separation or a second planet lying near the Einstein ring, but it is difficult to claim a secure detection due to the marginal fit improvement, lack of consistency among different data sets, and difficulty in uniquely specifying the nature of the tertiary component.

Results. With the observables of the event, it is estimated that the masses of the planet and host are $\sim\left(6.9 M_{\oplus}, 0.75 M_{\odot}\right)$ according to one solution and $\sim\left(2.8 M_{\oplus}, 0.80 M_{\odot}\right)$ according to the other solution, indicating that the planet is a super Earth around a K-type star, regardless of the solution. The fact that 16 , including the one reported in this work, out of 19 microlensing planets with $M \lesssim 10 M_{\oplus}$ were detected during the last 6 years well demonstrates the importance of high-cadence global surveys in detecting very low-mass planets.
\end{abstract}

Key words. gravitational microlensing - planets and satellites: detection

\section{Introduction}

The current microlensing surveys differentiate themselves from those of the previous era by their greatly increased observational cadence. High-cadence surveys with multiple observations per night became possible with the combination of the instrumental upgrade of existing surveys and the inauguration of a new survey employing globally distributed multiple telescopes equipped with cameras yielding a very large field of view (FOV). The Microlensing Observations in Astrophysics survey (MOA: Bond et al. 2001), which originally started its first phase experiment with the use of a $0.61 \mathrm{~m}$ telescope and a camera having a $1.3 \mathrm{deg}^{2} \mathrm{FOV}$, entered into its second phase by employing a $1.8 \mathrm{~m}$ telescope and replacing its old camera with a new one having a $2.2 \mathrm{deg}^{2}$ FOV. The Optical Gravitational Lensing Experiment (OGLE), which started its first-phase experiment with a $1.0 \mathrm{~m}$ telescope, is now in its fourth phase (OGLE-IV: Udalski et al. 2015) using a $1.3 \mathrm{~m}$ telescope mounted with a mosaic camera yielding a $1.4 \mathrm{deg}^{2}$ FOV. The Korea Microlensing Telescope Network (KMTNet: Kim et al. 2016) started its full operation in 2016 using its three $1.6 \mathrm{~m}$ telescopes, each of which is equipped with a camera providing a $4 \mathrm{deg}^{2} \mathrm{FOV}$. With the instrumental upgrade of the existing experiments and the advent of a new survey, the cadence of the current surveys reaches down to $<8$ minutes, which is two orders of magnitude higher than that of earlier experiments.

The increased survey area combined with the increased observational cadence resulted in the dramatic rise in the detection 


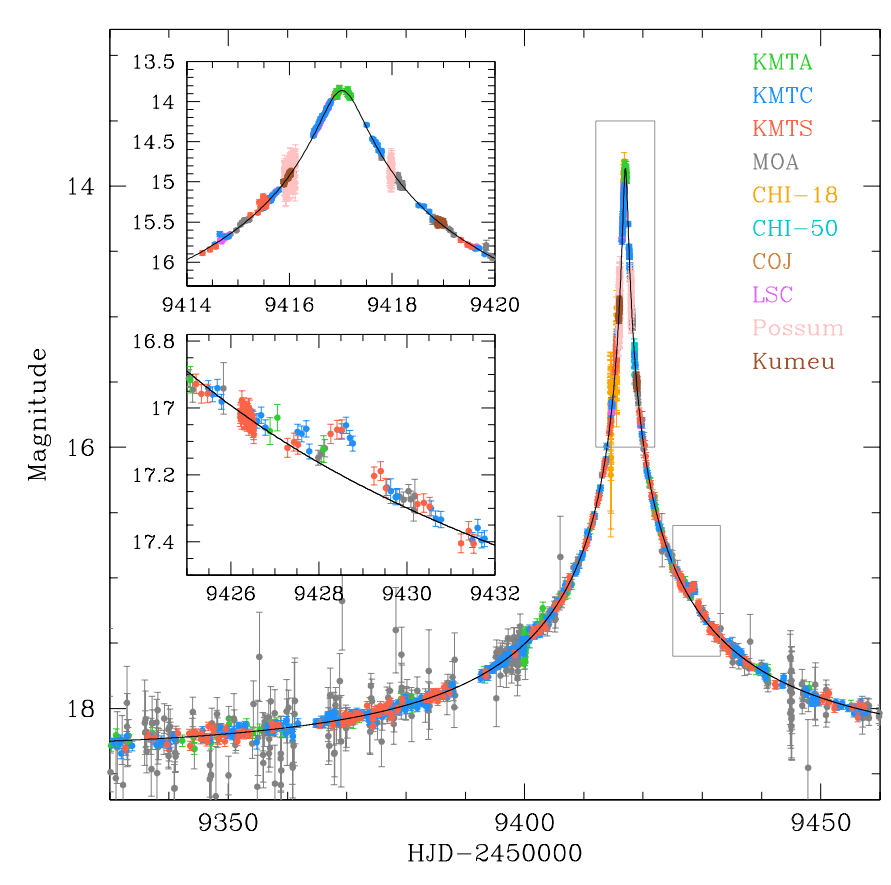

Fig. 1. Light curve of KMT-2021-BLG-0912. The insets show the zoom-in views of the peak and anomaly regions. The curve drawn over data points is a model based on the single-lens single-source interpretation.

rate of lensing events. The annual event rate, which was of the order of dozens in the 1990s and hundreds in the 2000s, has soared to more than 3000 from the combination of the three lensing surveys. The increase of the total event rate is accompanied by the rapid increase in the number of microlensing planet detections, and about 20 planetary systems are annually being reported.

Another important achievement of high-cadence global surveys is that they expand the channel of planet detections. In general, microlensing signals of planets appear as short-term anomalies on the lensing light curves produced by hosts. The duration of the planetary signal becomes shorter in proportion to the square root of the planet-to-host mass ratio $q$, that is, $t_{\text {planet }} \sim q^{1 / 2} t_{\mathrm{E}}$, where $t_{\mathrm{E}}$ represents the event time scale. Then the duration is several days for giant planets with $q \sim(O) 10^{-3}$ and less than one day ${ }^{1}$ for planets with $q \lesssim$ $(O) 10^{-4}$. As a result, it was difficult to detect planets with very low mass ratios from observations conducted in a surveyonly mode with the $\sim 1$ day cadence of the earlier generation surveys. To complement the inadequate cadence of the previous surveys, planetary lensing experiments had been carried out in a survey+followup mode, in which survey experiments focused mostly on event detections and followup experiments coordinated dense global coverage of the events detected by the survey experiments, for example, OGLE-2005-BLG-071Lb (Udalski et al. 2005; Dong et al. 2009) and OGLE-2005-BLG390Lb (Beaulieu et al. 2006). Dense followup observations require alerts of events with ongoing anomalies, but such observations were difficult for events with very short planetary signals due to the difficulty of predicting the time of the anomaly and immediately conducting followup observations. As a result, observations in the survey+followup mode were conducted most successfully for a special type of events with very high magni-

\footnotetext{
${ }_{1}^{1}$ In practice, longer durations may be observed for oblique source trajectories, e.g., KMT-2020-BLG-0414 (Zang et al. 2021) and the planet presented here.
}

Table 1. Followup data

\begin{tabular}{lll}
\hline \hline \multicolumn{1}{c}{ Telescopes } & \multicolumn{1}{c}{ Epoch $\left(\mathrm{HJD}^{\prime}\right)$} & Aperture \\
\hline LCO COJ & $9416,9421,9424$ & $1.0 \mathrm{~m}$ \\
LCO LSC & $9414,9415,9416,9419$ & $1.0 \mathrm{~m}$ \\
CHI-18 & $9414,9415,9416$ & $0.18 \mathrm{~m}$ \\
CHI-50 & 9416,9418 & $0.5 \mathrm{~m}$ \\
$\mu$ FUN Possum & $9415,9416,9417,9418$ & $0.4 \mathrm{~m}$ \\
$\mu$ FUN Kumeu & $9415,9416,9418$ & $0.4 \mathrm{~m}$ \\
\hline
\end{tabular}

Notes. HJD' $\equiv$ HJD -2450000 .

fications, for which not only the time of the peak could be predicted with a reasonable precision but also the chance of planetary anomaly near the peak was high: the "central magnification channel" (Griest \& Safizadeh 1998). High-cadence surveys with globally distributed telescopes dispense with the need for alerts for dense coverage of planetary signals, and thus they expand the channel of planet detections to all events regardless of lensing magnifications, for example, OGLE-2018-BLG-0567Lb and OGLE-2018-BLG-0962Lb (Jung et al. 2021).

In this work, we report the discovery of a very low mass-ratio planet found from the analysis of a lensing event detected in the 2021 season. Despite the short duration and weakness, the planetary signal, which occurred well after the high-magnification peak, was clearly detected by the dense coverage of the lensing surveys. We test various interpretations to confirm the planetary origin of the anomaly.

We present the analysis according to the following organization. In Sect. 2, we give an explanation for the observations of the lensing event and the acquired data. In Sect. 3, we describe the characteristics of the light-curve anomaly and depict various models tested to explain the origin of the anomaly, including binary-lens and binary-source models. We detail the procedures of the modeling and present results. We additionally test the possibility that the lens is composed of three masses. The procedures of characterizing the source star and estimating the angular Einstein radius are depicted in Sect. 4. We estimate the physical parameters of the planetary system in Sect. 5, and we summarize results and conclude in Sect. 6.

\section{Observations and data}

The planet we report in this work was detected from the observations of the lensing event KMT-2021-BLG-0912/MOA-2021BLG-233. The source of the event is located in the Galactic bulge field with the equatorial coordinates (RA, Decl.) $)_{\mathrm{J} 2000}=$ $(17: 59: 17.08,-31: 59: 51.50)$, corresponding to the Galactic coordinates $(l, b)=\left(-1^{\circ} .142,-4^{\circ} .091\right)$. The apparent baseline magnitude of the source is $I_{\text {base }} \sim 18.31$ according to the KMTNet scale.

The magnification of the source flux caused by lensing was first found by the KMTNet survey on 2021-05-19 (HJD' $\equiv$ HJD $-2450000 \sim 9353)$ at its early stage of the lensing magnification. The event was independently found by the MOA survey 51 days after the KMTNet discovery, and it was labeled as MOA-2021-BLG-233. Hereafter, we refer to the event as KMT2021-BLG-0912 following the convention of designating a lensing event by the ID of the survey that first discovered the event. Data from the KMTNet survey were acquired using the three $1.6 \mathrm{~m}$ telescopes located at the Siding Spring Observatory (SSO) in Australia (KMTA), Cerro Tololo Inter-American Observatory (CTIO) in Chile (KMTC), and South African Astronomical Observatory (SAAO) in South Africa (KMTS). The MOA survey 

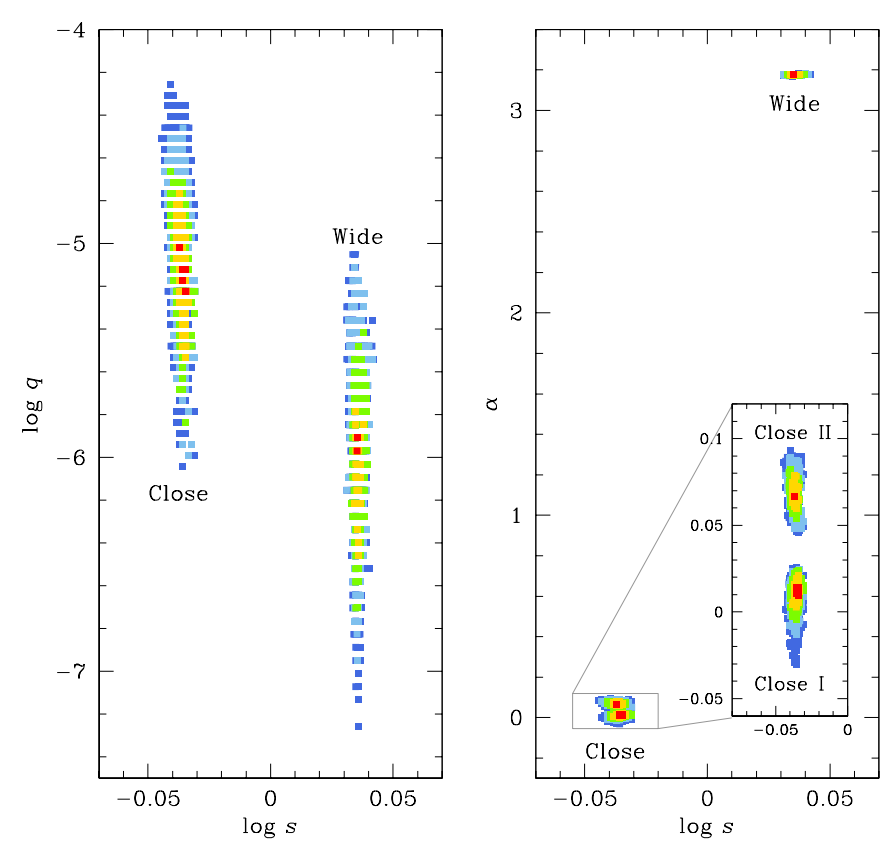

Fig. 2. $\Delta \chi^{2}$ map on the $\log s-\log q$ (left panel) and $\log s-\alpha$ (right panel) planes obtained from the 2L1S modeling. The maps shows three locals: close I $(\log s, \log q, \alpha) \sim(-0.04,-5.1,0.01)$, close II $\sim(-0.04,-5.1,0.07)$, and wide $\sim(0.04,-5.9,3.2)$ solutions. The inset in the right panel shows the zoom-in view of around the close I and close II solutions on the $\log s-\alpha$ (right panel) plane. The color coding is set to represents points with $\leq 1 n \sigma$ (red), $\leq 2 n \sigma$ (yellow), $\leq 3 n \sigma$ (green), $\leq 4 n \sigma$ (cyan), and $\leq 5 n \sigma$ (blue), where $n=2$.

utilized the $1.8 \mathrm{~m}$ telescope of the Mt. John Observatory in New Zealand. Images were mainly acquired in the $I$ band for the KMTNet survey and in the customized MOA- $R$ band for the MOA survey. For both surveys, a fraction of images were obtained in the $V$ band for the measurement of the source color. The event was also in the field of the OGLE survey. However, no OGLE observation was done because the OGLE telescope was shut down in the 2020 and 2021 seasons due to the Covid-19 pandemic.

The event was additionally covered by followup observations. At 2021 July 16, UT 21:30 (HJD' 9412.4), the KMTNet HighmagFinder system (Yang et al., in preparation) predicted that the event would reach a very high magnification based on the KMTNet data on the rising side of the light curve. Because the chance to detect planetary signals was high near the peak, the KMTNet group issued an alert for followup observations. In response to this alert, follow-up observations were conducted around the peak of the light curve using multiple telescopes that were globally distributed, including the two $1.0 \mathrm{~m}$ telescopes, one located at SSO (COJ) and the other at CTIO (LSC), of the Las Cumbres Observatory (LCO) global network, the $0.18 \mathrm{~m}$ and $0.5 \mathrm{~m}$ Newtonian telescopes located at El Sauce Observatory in Chile (CHI-18 and CHI-50), the two telescopes of the Microlensing Follow-Up Network ( $\mu$ FUN) group (Possum $0.4 \mathrm{~m}$ and Kumeu $0.4 \mathrm{~m}$ ) located in New Zealand. In Table 1, we list the follow-up telescopes and the epochs of observations. Furthermore, the KMTNet survey increased its observational cadence by employing the "auto-followup" system, which began its operation in the 2021 season for dense coverage of highmagnification events. However, even with the dense coverage by the combined data from the survey and follow-up observations, there is no significant anomaly over the peak of the light curve,

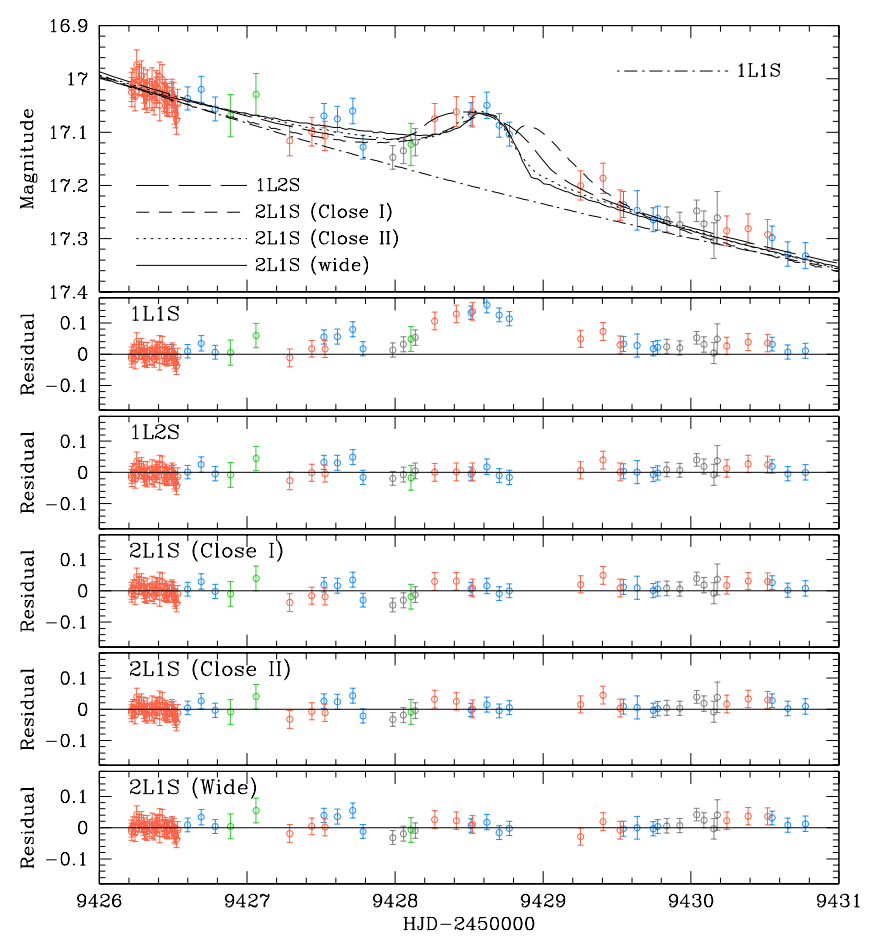

Fig. 3. Models curves and residuals of the five tested solutions, including the $1 \mathrm{~L} 1 \mathrm{~S}, 1 \mathrm{~L} 2 \mathrm{~S}$, three $2 \mathrm{~L} 1 \mathrm{~S}$ (close I, close II, and wide) solutions.

although these observations do help constrain the finite source effect. The zoom-in view around the peak region is shown in the upper inset of Figure 1. In Sect. 3.3, we will give an in-depth discussion of a possible central anomaly.

A short-term anomaly occurring much later (HJD' 9428.5) was noticed by C. Han from a revisit of the event on August 19 (HJD' $~ 9437$ ), when the source brightness was declining. The zoom-in view around the anomaly is presented in the lower inset of Figure 1. The anomaly, which was covered mostly by the KMTC and KMTS data sets, lasted for $\sim 1$ day centered at $\mathrm{HJD}^{\prime} \sim 9428.5$. At the peak of the anomaly, the data points deviate from the 1L1S model by $\sim 0.2 \mathrm{mag}$. After the anomaly, the event gradually returned to the baseline.

The data obtained by the KMTNet and MOA surveys were processed utilizing the pipelines of the individual groups: Albrow et al. (2009) for the KMTNet survey and Bond et al. (2001) for the MOA survey. These pipelines are developed based on the difference image technique (Tomaney \& Crotts 1996; Alard \& Lupton 1998). The followup data from the LCO, CHI-18, and CHI-50 observations were reduced using the ISIS pipeline (Alard 2000; Zang et al. 2018), and those from the $\mu$ FUN observations were reduced using the DoPHOT code (Schechter et al. 1993). For the individual data sets, the error bars estimated from the photometry pipelines were readjusted to take into consideration the scatter of data and to make the $\chi^{2}$ per degree of freedom for each data set unity following the routine described in Yee et al. (2012).

Figure 1 shows the light curve of KMT-2021-BLG-0912. The curve drawn over the data points is a $1 \mathrm{~L} 1 \mathrm{~S}$ model with lensing parameters $\left(t_{0}, u_{0}, t_{\mathrm{E}}\right) \sim\left(9417.02,6.0 \times 10^{-3}, 70\right.$ days $)$. Here $t_{0}$ is the time, expressed in HJD', of the closest lens-source approach and $u_{0}$ is the separation at $t_{0}$. The two small boxes drawn over the light curve indicate the regions around the peak and the anomaly. The magnification at the peak was $A_{\text {peak }} \sim 1 / u_{0} \sim 170$. 
Table 2. Close I 2L1S model parameters

\begin{tabular}{lll}
\hline \hline \multicolumn{1}{c}{ Parameter } & \multicolumn{1}{c}{ Standard } & \multicolumn{1}{c}{ Higher-order } \\
\hline$\chi^{2}$ & 1637.2 & 1601.9 \\
$t_{0}\left(\mathrm{HJD}^{\prime}\right)$ & $9417.020 \pm 0.002$ & $9417.020 \pm 0.003$ \\
$u_{0}\left(10^{-2}\right)$ & $0.60 \pm 0.02$ & $0.62 \pm 0.02$ \\
$t_{\mathrm{E}}(\mathrm{days})$ & $69.62 \pm 1.59$ & $69.53 \pm 2.31$ \\
$s$ & $0.920 \pm 0.002$ & $0.882 \pm 0.015$ \\
$q\left(10^{-5}\right)$ & $0.84 \pm 0.17$ & $2.77 \pm 0.67$ \\
$\alpha(\mathrm{rad})$ & $0.009 \pm 0.003$ & $0.083 \pm 0.012$ \\
$\rho\left(10^{-3}\right)$ & $2.47 \pm 0.37$ & $3.85 \pm 0.62$ \\
$\pi_{\mathrm{E}, N}$ & & $0.58 \pm 0.15$ \\
$\pi_{\mathrm{E}, E}$ & & $-0.11 \pm 0.06$ \\
$d s / d t\left(\mathrm{yr}^{-1}\right)$ & & $1.19 \pm 0.50$ \\
$d \alpha / d t\left(\mathrm{yr}^{-1}\right)$ & & $-1.76 \pm 0.37$ \\
\hline
\end{tabular}

Notes. HJD' $\equiv$ HJD -2450000 .

\section{Interpreting the event}

The light curve of the lensing event KMT-2021-BLG-0912 is characterized by a short-lasting anomaly on the otherwise smooth and symmetric 1L1S curve. This is a characteristic pattern of an event produced by a lens with a very low-mass companion (Gould \& Loeb 1992). Therefore, we first model the light curve under a binary-lens single-source (2L1S) interpretation. We additionally test a single-lens binary-source (1L2S) interpretation because it is known that a subset of 1L2S events can generate short-term anomalies in lensing light curves (Gaudi 1998; Gaudi \& Han 2004).

Considering an additional lens or source component in lensing modeling requires one to include extra parameters in addition to the $1 \mathrm{~L} 1 \mathrm{~S}$ lensing parameters, that is, $\left(t_{0}, u_{0}, t_{\mathrm{E}}\right)$. For a $2 \mathrm{~L} 1 \mathrm{~S}$ model, these extra parameters are $(s, q, \alpha)$, which represent the separation and mass ratio between the lens components, $M_{1}$ and $M_{2}$, and the angle between the source motion and the binary lens axis (source trajectory angle), respectively. For a 1L2S model, the extra parameters are $\left(t_{0,2}, u_{0,2}, q_{F}\right)$, which represent the time and separation at the closest approach of the source companion to the lens, and the flux ratio between the primary and secondary source components, $S_{1}$ and $S_{2}$. In the 1L2S modeling, we designate the parameters related to $S_{1}$ as $\left(t_{0,1}, u_{0,1}\right)$ to distinguish them from those related to $S_{2}$. For all tested models, we additionally include the parameter $\rho$, which represents the ratio of the angular source radius $\theta_{*}$ to the Einstein radius $\theta_{\mathrm{E}}$, that is, $\rho=\theta_{*} / \theta_{\mathrm{E}}$. This parameter (normalized source radius) is needed to account for finite-source effects that affect lensing light curves during the source passage over the lens or caustics.

In the modeling, we search for the set of lensing parameters (solution) that best describes the observed data. The 2L1S solution is investigated in two steps, in which we search for the binary parameters $(s, q)$ using a grid approach in the first step, and then refine the individual local solutions found from the first step by allowing all parameters to vary in the second step. For the searches of the parameters other than the grid parameters, we use a downhill approach. We adopt this two-step procedure to identify possible degenerate solutions, in which different combinations of lensing parameters result in similar lensing light curves. In the 1L2S modeling, we search for the solution using a downhill approach with initial values of $\left(t_{0,2}, u_{0,2}, q_{F}\right)$ assigned considering the time and strength of the anomaly. For the downhill approach, we use the Markov Chain Monte Carlo (MCMC) algorithm.
Table 3. Close II 2L1S model parameters

\begin{tabular}{lll}
\hline \hline \multicolumn{1}{c}{ Parameter } & \multicolumn{1}{c}{ Standard } & \multicolumn{1}{c}{ Higher-order } \\
\hline$\chi^{2}$ & 1626.9 & 1592.4 \\
$t_{0}\left(\mathrm{HJD}^{\prime}\right)$ & $9417.023 \pm 0.002$ & $9417.025 \pm 0.002$ \\
$u_{0}\left(10^{-2}\right)$ & $0.63 \pm 0.02$ & $0.62 \pm 0.03$ \\
$t_{\mathrm{E}}($ days $)$ & $67.38 \pm 1.45$ & $68.00 \pm 2.67$ \\
$s$ & $0.918 \pm 0.002$ & $0.914 \pm 0.014$ \\
$q\left(10^{-5}\right)$ & $1.21 \pm 0.20$ & $1.05 \pm 0.46$ \\
$\alpha(\mathrm{rad})$ & $0.072 \pm 0.004$ & $0.104 \pm 0.008$ \\
$\rho\left(10^{-3}\right)$ & $3.41 \pm 0.51$ & $2.68 \pm 0.63$ \\
$\pi_{\mathrm{E}, N}$ & & $0.15 \pm 0.28$ \\
$\pi_{\mathrm{E}, E}$ & & $0.00 \pm 0.09$ \\
$d s / d t\left(\mathrm{yr}^{-1}\right)$ & & $0.13 \pm 0.46$ \\
$d \alpha / d t\left(\mathrm{yr}^{-1}\right)$ & & $-0.87 \pm 0.65$ \\
\hline
\end{tabular}

Table 4. Wide 2L1S model parameters

\begin{tabular}{lll}
\hline \hline \multicolumn{1}{c}{ Parameter } & \multicolumn{1}{c}{ Standard } & \multicolumn{1}{c}{ Higher-order } \\
\hline$\chi^{2}$ & 1623.8 & 1611.6 \\
$t_{0}\left(\mathrm{HJD}^{\prime}\right)$ & $9417.023 \pm 0.002$ & $9417.027 \pm 0.003$ \\
$u_{0}\left(10^{-2}\right)$ & $0.59 \pm 0.02$ & $0.60 \pm 0.02$ \\
$t_{\mathrm{E}}($ days $)$ & $68.41 \pm 1.55$ & $67.78 \pm 1.59$ \\
$s$ & $1.089 \pm 0.002$ & $1.109 \pm 0.020$ \\
$q\left(10^{-5}\right)$ & $0.12 \pm 0.04$ & $0.17 \pm 0.40$ \\
$\alpha(\mathrm{rad})$ & $3.177 \pm 0.001$ & $3.177 \pm 0.032$ \\
$\rho\left(10^{-3}\right)$ & $0.88 \pm 0.21$ & $1.01 \pm 0.09$ \\
$\pi_{\mathrm{E}, N}$ & & $-0.06 \pm 0.29$ \\
$\pi_{\mathrm{E}, E}$ & & $0.11 \pm 0.08$ \\
$d s / d t\left(\mathrm{yr}^{-1}\right)$ & & $-0.56 \pm 0.63$ \\
$d \alpha / d t\left(\mathrm{yr}^{-1}\right)$ & & $-0.15 \pm 0.75$ \\
\hline
\end{tabular}

\subsection{Binary-lens (2L1S) interpretation}

According the planetary 2L1S interpretation, the planet-host separation $s$ can be heuristically estimated from the location of the anomaly in the lensing light curve. Under the approximation of a very low-mass companion, the planetary caustic induced by a planet lies at the location with a separation from the primary lens of

$u_{\mathrm{a}} \sim s-\frac{1}{s}$,

which has a negative value for a close binary with $s<1.0$ and a positive value for a wide binary with $s>1.0$ (Griest \& Safizadeh 1998; Han et al. 2006). Then, the planet-host separation is obtained by solving Equation (1) with respect to $s$, that is,

$s=\frac{1}{2}\left[u_{\mathrm{a}}+\left(u_{\mathrm{a}}^{2}+4\right)^{1 / 2}\right]$.

The $u_{\mathrm{a}}$ value corresponds to the source separation (from $M_{1}$ ) at the time of the anomaly, $t_{\mathrm{a}}$, and it is related to the lensing parameters by

$u_{\mathrm{a}}=\left[\left(\frac{t_{\mathrm{a}}-t_{0}}{t_{\mathrm{E}}}\right)^{2}+u_{0}^{2}\right]^{1 / 2}$.

With the time of the anomaly at $t_{\mathrm{a}} \sim 9428.5$ and the 1L1S lensing parameters, $\left(t_{0}, u_{0}, t_{\mathrm{E}}\right) \sim\left(9417.02,6.0 \times 10^{-3}, 70\right.$ days $)$, the source separation is $u_{\mathrm{a}} \sim \pm 0.16$. Then, there exist two solutions of the planet separations of

$s_{c} \sim 0.92 \quad$ and $\quad s_{w} \sim 1.09$,

where the former and latter values correspond to the close and wide solutions, respectively. 

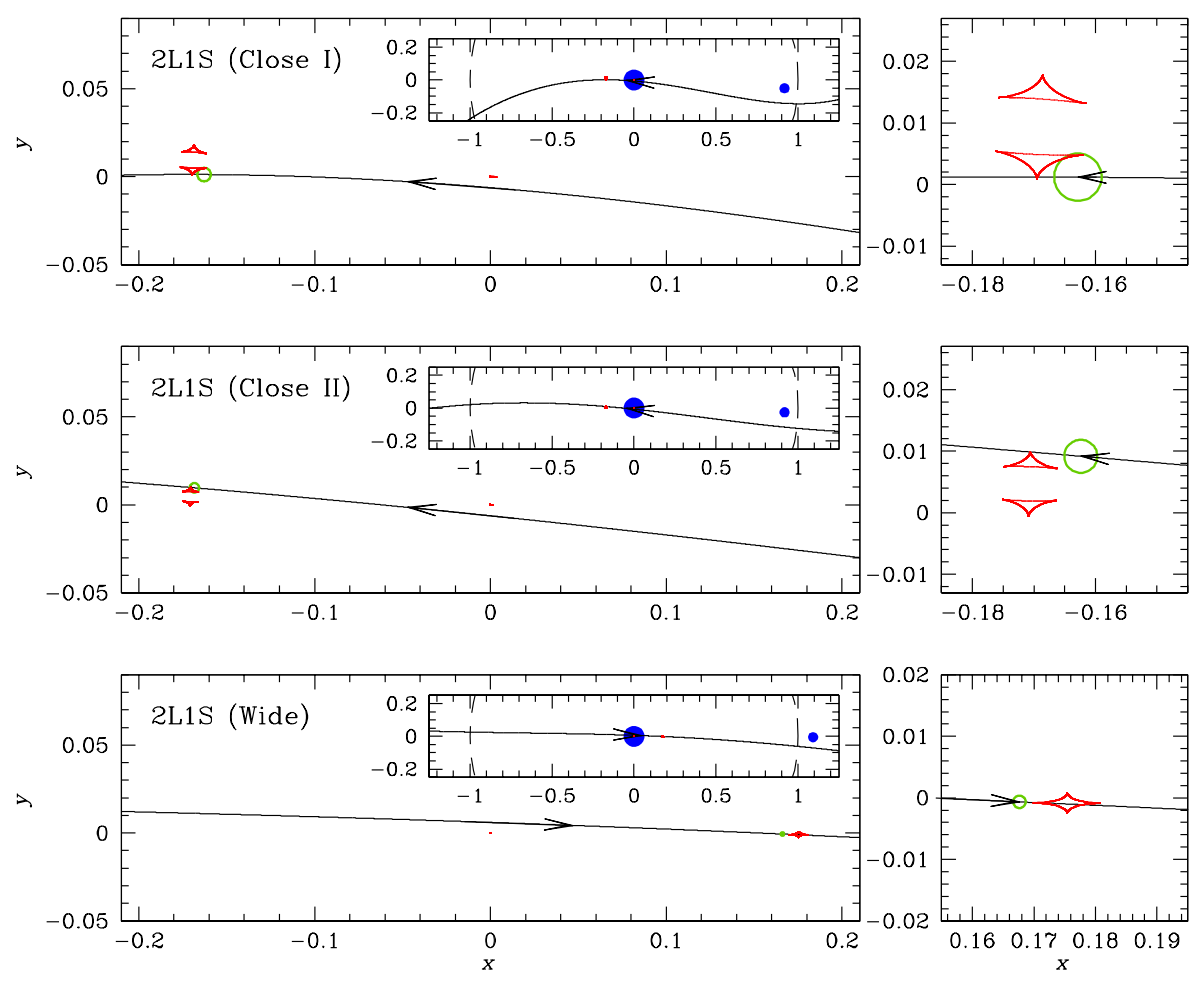

Fig. 4. Lens system configurations of the three 2 L1S solutions: close I, close II, wide solutions. In each panel, the small figures composed of concave curves represent the caustics and the curve with an arrow denotes the source trajectory. For each solution, the inset of the left panel shows the whole view including the lens positions (marked by blue dots) and the Einstein ring (dashed circle). The panel on the right side shows the zoom-in view around the planetary caustic. The green empty circle on the source trajectory represents the source for which the size is scaled to the caustic size. Lengths are scaled to the Einstein radius and the coordinates are centered at the position of the primary of the planetary system. The lensing parameters corresponding to the individual solutions are listed in Tables $2-4$.

From the 2L1S modeling, we identify 3 local solutions. Figure 2 shows the locations of these local solutions in the $\chi^{2}$ map on the $\log s-\log q$ (left panel) and $\log s-\alpha$ (right panel) planes. The binary lensing parameters of the individual solutions are $(\log s, \log q, \alpha) \sim(-0.04,-5.1,0.01), \sim(-0.04,-5.1,0.07)$, and $\sim(0.04,-5.9,3.18)$ in logarithmic scale and radians, or $(s, q, \alpha) \sim\left(0.9,0.9 \times 10^{-5}, 0.6^{\circ}\right), \sim\left(0.9,0.9 \times 10^{-5}, 4.0^{\circ}\right)$, and $\sim\left(1.1,0.13 \times 10^{-5}, 182.2^{\circ}\right)$ in linear scale and degrees. We refer to the individual solutions as "close I", "close II", and "wide" solutions, respectively. We note that the $s$ and $q$ values for the two close solutions are similar to each other, but they have slightly different values of the source trajectory angle, as shown in the inset of the right panel. The binary separations of the close, $s_{c}$, and wide solution, $s_{w}$, match well the heuristically estimated values in Equation (4). We note that although the binary separations $s_{c}$ and $s_{w}$ are approximately in the relation of $s_{c} \sim 1 / s_{w}$, the degeneracy between the close and wide solutions has a different origin from the close-wide degeneracy between a pair of solutions for central planet-induced anomalies appearing in highmagnification events (Griest \& Safizadeh 1998; Dominik 1999). We will discuss in detail the origins of the degeneracies below. Regardless of the solutions, the estimated mass ratios are very small, of the order of $10^{-5}$ for the close solutions or even smaller for the wide solutions, indicating that the companion to the lens is a planetary-mass object with a very small planet-to-host mass ratio according to the $2 \mathrm{~L} 1 \mathrm{~S}$ interpretation.

The model curves and residuals of the individual 2L1S solutions around the region of the anomaly are shown in Figure 3, and their lensing parameters are listed in Tables $2-4$ together with the $\chi^{2}$ values of the fits. For each solution, we present two sets of solutions, in which one solution is obtained under the assumption of a rectilinear relative lens-source motion, standard model, and the other solution is obtained by considering the deviation from the rectilinear motion caused by the orbital motions of Earth (microlens-parallax effect: Gould 1992) and the binary lens (lens-orbital effect: Dominik 1999), higher-order model. We will further discuss these higher-order effects below. From the comparison of the solutions, it is found that the close II solution provides the best fit to the data, but the $\chi^{2}$ differences from the other solutions, $\Delta \chi^{2}=9.5$ with respect to the close I solution and 19.2 with respect to the wide solution, are relatively modest. As a rule of thumb, differences of $\Delta \chi^{2}>10$ are required to distinguish between solutions with reasonable confidence, but careful consideration must be given when the differences are not much larger, as in the present case.

Figure 4 shows the configurations of the lens systems for the three degenerate 2L1S solutions. In each panel, the small closed figures composed of concave curves represent the caustics induced by the planet, and the curve with an arrow represents the source trajectory. The configurations correspond to the models obtained with the consideration of the higher-order effects, and thus the source trajectory is slightly curved due to the microlensparallax effect. The caustic varies in time due to the lens orbital motion, and the presented caustics correspond to the ones at the time of the anomaly. The planetary companion induces two sets of caustics, in which one is located very close to the host of the planet (central caustic) and the other is located away from the host (planetary caustic). The planetary caustic induced by a close 

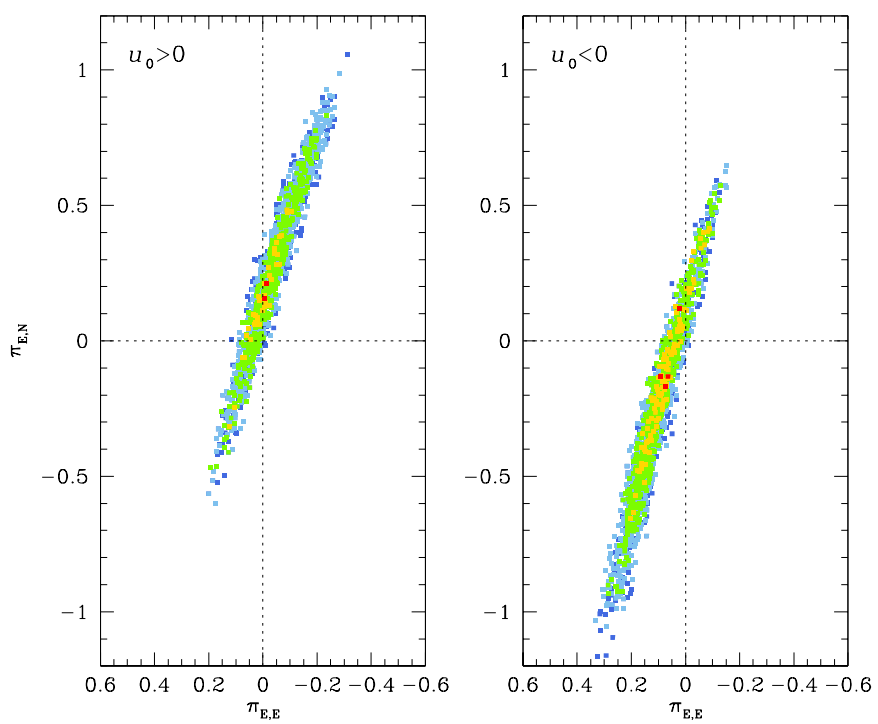

Fig. 5. Scatter plot of MCMC points on the $\pi_{\mathrm{E}, N}-\pi_{\mathrm{E} E}$ parameter plane. Color coding is same as that in Fig. 2, except that $n=1$. The right and left panels are the plots for the two degenerate solutions with $u_{0}>0$ and $u_{0}<0$ solutions, respectively.

planet is located on the opposite side of the planet with respect to the host and is composed of two sets of closed curves, while the planetary caustic induced by a wide planet is located on the planet side and is composed of a single closed curve (Han et al. 2006). According to the close I and II solutions, the anomaly was produced by the source passage over the upper and lower planetary caustics induced by a close planet, respectively. According to the wide solution, on the other hand, the anomaly was generated by the source passage over the planetary caustic of a wide planet. According to all 2L1S solutions, the source did not cross the central caustic. As a result, the central region does not exhibit an obvious anomalous feature, but we will discuss possible central anomalies in Sect. 3.3.

The form of the degeneracy among the three $2 \mathrm{~L} 1 \mathrm{~S}$ solutions is very rare because it arises from the special configuration of the lens system. The degeneracy between the close I and II solutions, which we refer to as the "upper-lower degeneracy", occurs due to the special lens-system configuration, in which the source moves almost in parallel with the planet-host axis and passes through the upper and lower parts of the planetary caustic with similar patterns of magnification excess. The degeneracy between the close and wide solutions, which we referred to as the "close-wide degeneracy for planetary caustics", arises due to the small caustic size caused by the very low planet/host mass ratio. In this case, it is difficult to delineate the detailed structure of the anomaly not only because of the short duration caused by the low mass ratio but also because of the featureless structure caused by finite-source effects. This degeneracy is different from the closewide degeneracy for central anomalies, arising from the intrinsic similarity between the central caustics induced by a close and a wide planet, in the sense that the anomaly was produced by planetary caustics. The planetary caustics induced by a close and a wide planet differ both in shape and number, resulting in different patterns of magnification excess (Chung et al. 2005). Then, the close-wide degeneracy for planetary caustics is an accidental degeneracy arising from the inadequate precision and cadence of observations for very short anomalies induced by planets with very low mass ratios.

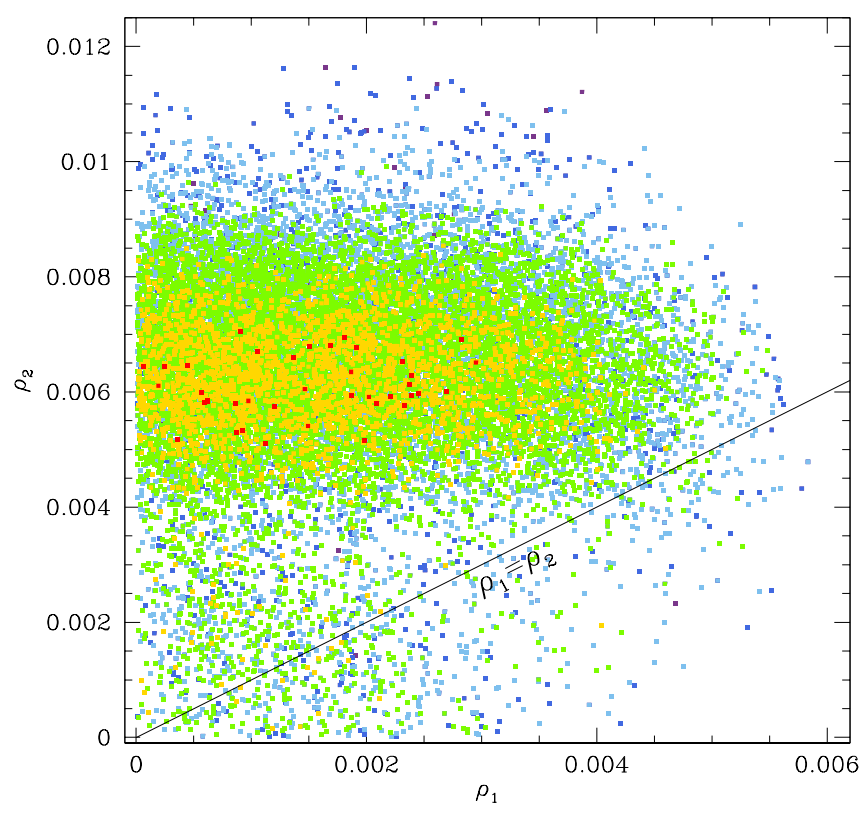

Fig. 6. Scatter plot of points in the MCMC chain on the $\rho_{1}-\rho_{2}$ plane for the 1L2S model. Color coding is the same as that in Fig. 5. The black line represents the equation $\rho_{1}=\rho_{2}$.

We check the feasibility of measuring the additional observables of the angular Einstein radius $\theta_{\mathrm{E}}$ and microlens parallax $\pi_{\mathrm{E}}$, which can constrain the mass and distance to the lens. The prerequisite for the estimation of $\theta_{\mathrm{E}}$ is the measurement of the normalized source radius $\rho$, because the Einstein radius is estimated by

$\theta_{\mathrm{E}}=\frac{\theta_{*}}{\rho}$,

where the angular source radius $\theta_{*}$ can be estimated from the color and brightness of the source. We will discuss the detailed procedure of $\theta_{*}$ measurement in Sect. 4. The $\rho$ value is measured by analyzing the planetary anomaly that is affected by finitesource effects, and the values corresponding to the individual 2L1S solutions are listed in Tables $2-4$. We note that the $\rho$ values vary in the range of $[0.88-3.41] \times 10^{-3}$ depending on the solutions.

The $\pi_{\mathrm{E}}$ value is measured from the deviation in the light curve caused by the microlens-parallax effect. For the measurement of $\pi_{\mathrm{E}}$, we conduct additional sets of modeling for the individual $2 \mathrm{~L} 1 \mathrm{~S}$ solutions by considering the parallax effect. Because it is known that the orbital motion of the lens can result in similar deviation to that induced by the parallax effect (Batista et al. 2011), we simultaneously consider both the microlens-parallax and lens-orbital effects (Dominik 1999) in the modeling. For the consideration of these higher-order effects, we add four extra parameters in the modeling: $\left(\pi_{\mathrm{E}, N}, \pi_{\mathrm{E}, E}\right)$ for the microlens parallax effect and $(d s / d t, d \alpha / d t)$ for the lensorbital effect. The two parallax parameters denote the north and east components of the microlens-parallax vector $\pi_{\mathrm{E}}=$ $\left(\pi_{\text {rel }} / \theta_{\mathrm{E}}\right)(\boldsymbol{\mu} / \mu)$, respectively, and the two orbital parameters denote the annual change rates of the binary separation and source trajectory angle, respectively

The lensing parameters of the individual 2L1S solutions considering the higher-order effects are listed in Tables $2-4$. It is found that the fits of the higher-order models improve by $\Delta \chi^{2}=35.2,34.5$, and 12.2 with respect to the standard models 
Table 5. 1L2S model parameters

\begin{tabular}{ll}
\hline \hline Parameter & \multicolumn{1}{c}{ Value } \\
\hline$\chi^{2}$ & 1626.6 \\
$t_{0,1}\left(\mathrm{HJD}^{\prime}\right)$ & $9417.022 \pm 0.002$ \\
$u_{0,1}\left(10^{-2}\right)$ & $0.60 \pm 0.02$ \\
$t_{0,2}\left(\mathrm{HJD}^{\prime}\right)$ & $9428.575 \pm 0.067$ \\
$u_{0,2}\left(10^{-2}\right)$ & $0.22 \pm 0.13$ \\
$t_{\mathrm{E}}($ days $)$ & $67.91 \pm 1.70$ \\
$\rho_{1}\left(10^{-3}\right)$ & $<4.0$ \\
$\rho_{2}\left(10^{-3}\right)$ & $6.41 \pm 2.32$ \\
$q_{F}$ & $0.003 \pm 0.001$ \\
\hline
\end{tabular}

for the close I, close II, and wide solutions, respectively. We note that the uncertainties of the planet parameters $(s, q)$ are bigger than those estimated from the standard models, although the fits of the higher-order models improve. This is a general tendency in lens modeling arising because the relative motion of the source with respect to the caustic has more degree of freedom. Figure 5 shows the scatter plot of points in the MCMC chain on the $\pi_{\mathrm{E}, E^{-}}$ $\pi_{\mathrm{E}, N}$ parameter plane. The plot is based on the close II model, which yields the best fit among the solutions, and the other models result in similar scatter plots. It shows that the component of $\pi_{\mathrm{E}}$ that is parallel with the direction of Earth's acceleration, $\pi_{\mathrm{E}, \|}$, is well constrained, but the uncertainty of the perpendicular component, $\pi_{\mathrm{E}, \perp}$, is considerable. We also check the so-called ecliptic degeneracy between a pair of solutions with $u_{0}>0$ and $u_{0}<0$ arising due to the mirror symmetry of the source trajectory with respect to the binary axis (Skowron et al. 2011). The scatter plot for the $u_{0}<0$ solution is also presented in Figure 5. It is found that the $u_{0}<0$ solution results in a similar pattern to that of the $u_{0}>0$ solution.

\subsection{Binary-source (1L2S) interpretation}

We also check the interpretation in which the observed anomaly was produced by a companion to the source. For a lensing event involved with two source stars, the observed flux, $F$, is the superposition of fluxes from the events associated with the individual source stars (Griest \& Hu 1992; Han \& Gould 1997), that is,

$F=F_{1}\left(A_{1}+q_{F} A_{2}\right)$.

Here $F_{1}$ represents the flux of the primary source, and $A_{1}$ and $A_{2}$ denote the lensing magnifications associated with the individual source stars, that is,

$A_{i}=\frac{u_{i}^{2}+2}{u_{i}\left(u_{i}^{2}+4\right)^{1 / 2}} ; \quad u_{i}=\left[u_{0, i}^{2}+\left(\frac{t-t_{0, i}}{t_{\mathrm{E}}}\right)^{2}\right]^{1 / 2}$.

In Table 5, we list the lensing parameters obtained from the 1L2S modeling, and the model curve and residual around the anomaly are shown in Figure 3. The values $\rho_{1}$ and $\rho_{2}$ denote the normalized radii of the primary and companion source stars, respectively. It is found that the $1 \mathrm{~L} 2 \mathrm{~S}$ model also well describes the anomaly, yielding a fit that is comparable to those of the $2 \mathrm{~L} 1 \mathrm{~S}$ models.

Although the 1L2S model well describes the observed anomaly, it is rejected due to its unphysical lensing parameters. According to the model, the flux from the source companion comprises a very minor fraction, $q_{F} \sim 0.3 \%$, of the flux from the primary source, but the estimated normalized radius of the source companion, $\rho_{2} \sim 6.4 \times 10^{-3}$, is significantly greater than the upper limit of the primary source, $\rho_{1, \max } \sim 4.0 \times 10^{-3}$. This

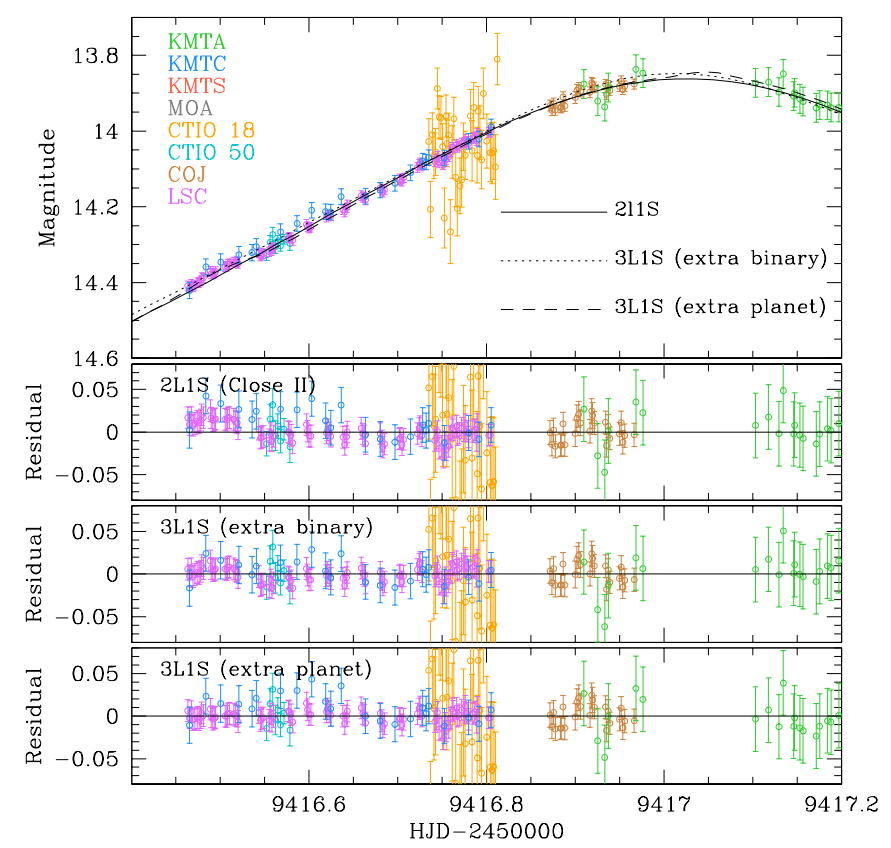

Fig. 7. Zoom-in view of the peak region. The curves drawn over the data points are the $2 \mathrm{~L} 1 \mathrm{~S}$ model (close II) and two 3L1S models. The lower panels shows the residuals from the individual models.

is shown in Figure 6, in which we plot the points in the MCMC chain on the $\rho_{1}-\rho_{2}$ plane. With the contradiction that the primary source is smaller than its very faint companion, we rule out the 1L2S interpretation of the event.

\subsection{Extra lens component: $3 L 1 S$ interpretation}

Although the peak region of the light curve does not exhibit an obvious anomalous feature, the region is susceptible to deviations induced by an extra lens component. This is because the event reached a very high magnification, implying that the source passed through the central magnification region, around which an extra lens component, if it exists, would induce a caustic affecting the magnification pattern of the region (Gaudi et al. 1998). We recall from Sect. 2 that the peak was subjected to high-cadence followup and auto-followup observations, which significantly enhance the sensitivity to low-amplitude anomalies. Therefore, we inspect small deviations in the peak region.

The peak region of the light curve is shown in top panel of Figure 7. The solid curve drawn over the data points is the model curve of the 2L1S (close II) solution, and the residual from the model is shown in the second panel. It is found that the model leaves small deviations with $\lesssim 0.03$ mag level mainly in the LSC data set taken during $9416.46 \lesssim \mathrm{HJD}^{\prime} \lesssim 9416.80$. Although the deviation is subtle, we check the possibility of a third lens component, $M_{3}$, for the origin of the deviation.

The lensing modeling with three lens components (3L1S model) requires one to include extra parameters in addition to those of the $2 \mathrm{~L} 1 \mathrm{~S}$ modeling. These parameters are $\left(s_{3}, q_{3}, \psi\right)$, which represent the separation and mass ratio between $M_{1}$ and $M_{3}$, and the orientation angle of $M_{3}$ with respect to the $M_{1}$ $M_{2}$ axis with a center at the position of $M_{1}$, respectively. The 3L1S modeling is done in two steps, in which we conduct grid searches for the parameters related to $M_{3}$, that is, $\left(s_{3}, q_{3}, \psi\right)$, in the first step, and then refine the local solutions found from the grid search by allowing all parameters to vary. In the grid search, 


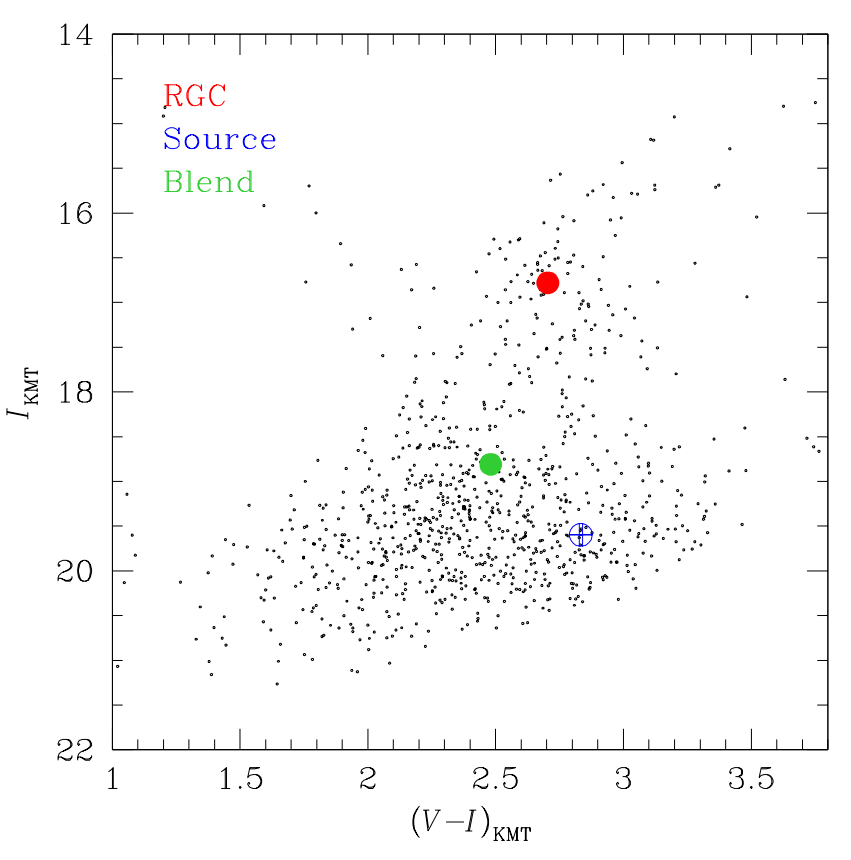

Fig. 8. Locations of the source, blend, and RGC centroid in the instrumental CMD of stars around the source.

we set the other parameters by adopting those of the 2L1S solution (close II solution) under the assumption that the anomaly induced by a tertiary lens component would be confined in a small region around the peak, and thus could be treated as a perturbation (Bozza 1999; Han et al. 2001).

From the 3L1S modeling, we find two sets of local solutions. According to one set, the peak residual is reduced by a low-mass binary companion with a projected separation either much bigger or smaller than the Einstein radius ("extra-binary" solution), and according to the other solution, the residual is explained by a second planet with a separation similar to the Einstein radius ("extra-planet" solution). The triple-lens parameters of the solutions are

$\left(\log s_{3}, \log q_{3}, \psi\right) \sim \begin{cases}( \pm 0.68,-0.82,1.67), & \text { extra binary, } \\ (0.0,-5.21,2.01), & \text { extra planet. }\end{cases}$

Figure 7 shows the models curves and residuals of the extrabinary (for the wide solution with $s \sim 4.8$ ) and extra-planet 3L1S solutions. Although the improvement of the fit in the peak region supports the possibility of an extra lens component, it is difficult to claim a secure detection of a tertiary lens component due to several reasons. First, the fit improvement is marginal: $\Delta \chi^{2} \sim 52.2$ and 34.6 with respect to the 2L1S solution for the extra binary and planet solutions, respectively. Second, the two data sets covering the peak anomaly, LSC and KMTC data sets, do not show a strong consistency as shown in the bottom two panels of Figure 7. Third, there exist multiple solutions, that is, extra-binary and extra-planet solutions, that can explain the central residual, making it difficult to uniquely specify the nature of the extra lens component even if the signal is real.

\section{Source star and Einstein radius}

In this section, we estimate the angular source radius in order to estimate the angular Einstein radius using the relation in Equation (5). We deduce $\theta_{*}$ from the color and magnitude of the source star. For the measurement of the reddening and extinction-corrected (dereddened) color and magnitude,
Table 6. Angular Einstein radius and relative lens-source proper motion

\begin{tabular}{lcl}
\hline \hline \multicolumn{1}{c}{ Model } & $\theta_{\mathrm{E}}(\mathrm{mas})$ & $\mu\left(\mathrm{mas} \mathrm{yr}^{-1}\right)$ \\
\hline Close I & $0.49 \pm 0.09$ & $2.57 \pm 0.478$ \\
Close II & $0.70 \pm 0.18$ & $3.76 \pm 0.978$ \\
Wide & $1.85 \pm 0.20$ & $9.96 \pm 1.11$ \\
\hline
\end{tabular}

$(V-I, I)_{0}$, from uncalibrated values, $(V-I, I)$, in the instrumental color-magnitude diagram (CMD), we use the Yoo et al. (2004) method, which utilizes the centroid of red giant clump (RGC), for which its dereddened color and magnitude, $(V-I, I)_{\mathrm{RGC}, 0}$, are known, for calibration.

Figure 8 shows the locations of the source and RGC centroid in the instrumental CMD of stars around the source constructed using the pyDIA photometry (Albrow 2017) of the KMTC data. The values of $(V-I, I)$ are measured from regression of the $I$ and $V$-band KMTC data processed using the pyDIA code with the change of the event magnification. Also marked is the location of the blend with $(V-I, I)_{b}=(2.48,18.81)$. The measured instrumental color and magnitude are $(V-I, I)_{s}=$ $(2.833 \pm 0.006,19.597 \pm 0.001)$ for the source and $(V-I, I)_{\mathrm{RGC}}=$ $(2.704,16.780)$ for the RGC centroid. With the offsets in color and magnitude, $\Delta(V-I, I)$, between the source and RGC centroid together with the known dereddened color and magnitude of the RGC centroid, $(V-I, I)_{\mathrm{RGC}, 0}=(1.060,14.510)$ (Bensby et al. 2013; Nataf et al. 2013), the dereddened values of the source are estimated as

$$
\begin{aligned}
(V-I, I)_{s, 0} & =(V-I, I)_{\mathrm{RGC}, 0}+\Delta(V-I, I) \\
& =(1.189 \pm 0.006,17.327 \pm 0.001)
\end{aligned}
$$

indicating that the source is a bulge subgiant with a K4 spectral type. We convert the $V-I$ color into $V-K$ color using the relation of Bessell \& Brett (1988), and then interpolate the angular source radius from the $(V-K)-\theta_{*}$ relation of Kervella et al. (2004). The estimated source radius from this procedure is

$\theta_{*}=(1.87 \pm 0.13) \mu$ as.

With the measured value of $\theta_{*}$, the angular Einstein radius is estimated by Equation (5) and the relative lens-source proper motion is computed by $\mu=\theta_{\mathrm{E}} / t_{\mathrm{E}}$. In Table 6 , we list the estimated values of $\theta_{\mathrm{E}}$ and $\mu$ corresponding to the three degenerate $2 \mathrm{~L} 1 \mathrm{~S}$ solutions. We note that the value of the angular source radius does not vary depending on the solutions, but the values of $\theta_{\mathrm{E}}$ and $\mu$ have different values depending on the solutions because the individual solutions have different values of $\rho$.

We check whether a significant fraction of blended flux comes from the lens by measuring the astrometric offset between the image centroid at the baseline and the source position measured from the difference image. For the measurement of the source position, we use an image taken with the use of the $3.6 \mathrm{~m}$ Canada-France-Hawaii Telescope under a good seeing $\left(0^{\prime \prime} .65\right)$ condition at the time around the peak. The measured offset is

$\Delta \theta(N, E)=(26 \pm 17,-86 \pm 8)$ mas.

The offset is much bigger than the uncertainty, indicating that the major origin of the blended flux is not the lens. It must therefore be either a companion to the source, a companion to the lens, or an ambient star. Note that if the blended light is due to a star in or near the bulge, then its color and absolute magnitude $\left[(V-I)_{0}, M_{I}\right] \simeq(0.86,2.0)$ indicate that it is a subgiant. Based on the surface density of subgiants toward this field, the chance that an ambient subgiant would be projected within $\sim 100$ mas 


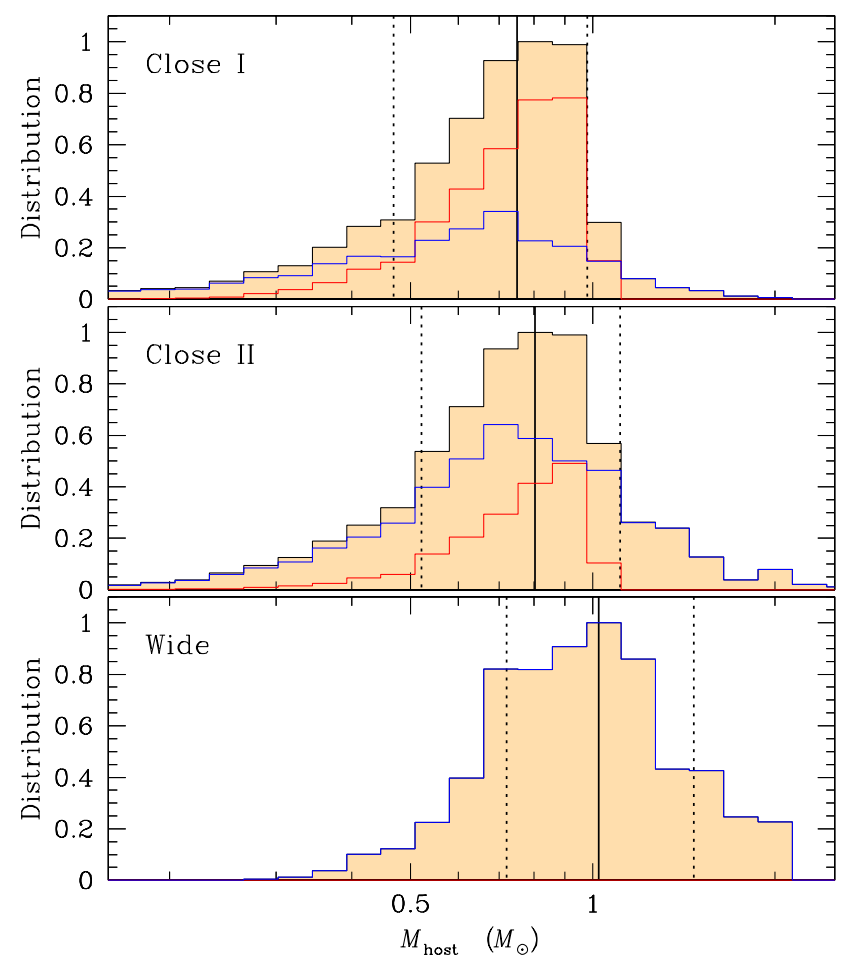

Fig. 9. Posterior distributions of the lens mass constructed from the Bayesian analyses based on the observables of the three $2 \mathrm{~L} 1 \mathrm{~S}$ solutions. For each distribution, the solid vertical line indicates the median, and the two dotted lines represent the $1 \sigma$ range of the distribution. The blue and red curves denote the contribution by the disk and bulge lens populations, and the solid distribution is the sum of the two populations.

of the lens is about $p \sim 6 \times 10^{-4}$. In addition, the probability that the source would have a subgiant companion at projected separation $\gtrsim 700 \mathrm{AU}$ is also relatively low, roughly $p \sim 3 \times 10^{-3}$. A similar probability applies to companions to the lens. Thus, we cannot reliably distinguish among these possibilities based on only the measured astrometric offset. The main impact of the blended light on the analysis is that it places an upper limit on the lens flux.

\section{Physical parameters}

We estimate the physical parameters of the planetary system using the observables of the lensing event. The mass and distance to the lens system can be uniquely determined with the observables of $\pi_{\mathrm{E}}$ and $\theta_{\mathrm{E}}$ using the relation

$$
M=\frac{\theta_{\mathrm{E}}}{\kappa \pi_{\mathrm{E}}} ; \quad D_{\mathrm{L}}=\frac{\mathrm{AU}}{\pi_{\mathrm{E}} \theta_{\mathrm{E}}+\pi_{\mathrm{S}}} .
$$

Here $\kappa=4 G /\left(c^{2} \mathrm{AU}\right)$ and $\pi_{\mathrm{S}}=\mathrm{AU} / D_{\mathrm{S}}$ denotes the parallax of the source located at a distance $D_{\mathrm{S}}$ (Gould 2000). For KMT2021-BLG-0912, the uncertainty of $\pi_{\mathrm{E}}$ is considerable, as shown in Figure 5, making it difficult to uniquely determine $M$ and $D_{\mathrm{L}}$ using the relation in Equation (12), but the physical lens parameters can still be constrained with the remaining observables $\theta_{\mathrm{E}}$ and $t_{\mathrm{E}}$, which are related to the physical parameters by

$t_{\mathrm{E}}=\frac{\theta_{\mathrm{E}}}{\mu} ; \quad \theta_{\mathrm{E}}=\left(\kappa M \pi_{\text {rel }}\right)^{1 / 2}$,

where $\pi_{\text {rel }}=\operatorname{AU}\left(1 / D_{\mathrm{L}}-1 / D_{\mathrm{S}}\right)$. We estimate the physical parameters by conducting a Bayesian analysis with the constraints

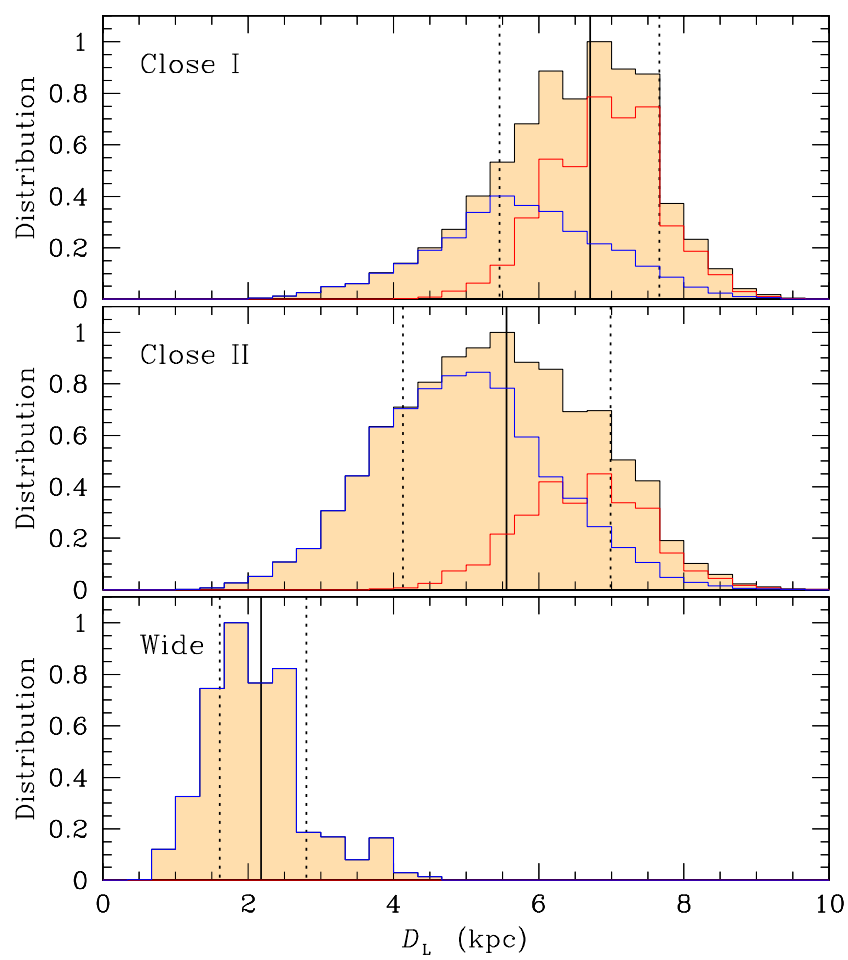

Fig. 10. Posterior distributions of the distance to the lens. Notations are same as those in Fig. 9.

Table 7. Physical parameters

\begin{tabular}{lccc}
\hline \hline Parameter & Close I & Close II & Wide \\
\hline$M_{\text {host }}\left(M_{\odot}\right)$ & $0.75_{-0.28}^{+0.23}$ & $0.80_{-0.28}^{+0.31}$ & $1.02_{-0.30}^{+0.45}$ \\
$M_{\text {planet }}\left(M_{\oplus}\right)$ & $6.92_{-2.59}^{+2.13}$ & $2.81_{-0.99}^{+1.08}$ & $0.58_{-0.17}^{+0.25}$ \\
$D_{\mathrm{L}}(\mathrm{kpc})$ & $6.71_{-1.25}^{+0.96}$ & $5.56_{-1.43}^{+1.43}$ & $2.18_{-0.57}^{+0.63}$ \\
$a_{\perp}(\mathrm{AU})$ & $3.03_{-2.46}^{+3.46}$ & $3.14_{-2.33}^{+3.94}$ & $3.81_{-2.81}^{+4.89}$ \\
\hline
\end{tabular}

provided by $\theta_{\mathrm{E}}$ and $t_{\mathrm{E}}$ and using the Galactic model defining the physical and dynamical distributions and mass function of Galactic objects. Although $\pi_{\mathrm{E}}$ is uncertain, we consider its constraint by imposing the restriction given by the covariance matrix of the parallax ellipse, that is, Figure 5. For the three degenerate solutions, the $t_{\mathrm{E}}$ values are similar to one another, but the $\theta_{\mathrm{E}}$ values vary substantially depending on the solutions, and thus we conduct three sets of analysis for the individual solutions.

The Bayesian analysis is carried out by producing a large number $\left(6 \times 10^{6}\right)$ of artificial lensing events from a Monte Carlo simulation using the Galactic model. We adopt the Jung et al. (2021) Galactic model that is constructed based on the Robin et al. (2003) and Han \& Gould (2003) models for the physical distributions of disk and bulge objects, Jung et al. (2021) and Han \& Gould (1995) models for the dynamical distribution for disk and bulge objects, and the Jung et al. (2018) mass function. In the mass function, we include brown-dwarf and white-dwarf populations of lenses, but exclude neutron stars and black holes because it is extremely unlikely that planets could remain bound after supernova explosions. For a more detailed description of the Galactic model, see Jung et al. (2021). With the artificial lensing events produced from the simulation, we construct the posterior distributions of $M$ and $D_{\mathrm{L}}$ for the events with observables consistent with the measured values. 
Figures 9 and 10 show the posterior distributions of $M_{\text {host }}=$ $M_{1}$, and $D_{\mathrm{L}}$, respectively. In Table 7 , we summarize the physical parameters of $M_{\text {host }}, M_{\text {planet }}=q M_{\text {host }}, D_{\mathrm{L}}$, and $a_{\perp}=s D_{\mathrm{L}} \theta_{\mathrm{E}}$ estimated based on the three 2L1S solutions. For each parameter, the representative value is chosen as the median value of the Bayesian distribution, and the lower and upper limits are estimated as $16 \%$ and $84 \%$ of the distribution. The host is a Ktype star according to the close solutions, while it is a G-type star according to the wide solution. The estimated mass of the host for the wide solution is bigger than those expected from the close solutions because the input value of the Einstein radius $\left(\theta_{\mathrm{E}} \sim 1.85\right.$ mas) for the wide solution is substantially bigger than those of the close solutions $\left(\theta_{\mathrm{E}} \sim 0.49\right.$ mas for the close I solution and $\sim 0.70$ mas for the close II solution). For the same reason, the estimated distance to the lens is closer for the wide solution than the distances estimated from the close solutions. The mass of the planet estimated from the close solutions $\left(M_{\text {planet }} \sim 6.9 M_{\oplus}\right.$ for the close I solution and $\sim 2.8 M_{\oplus}$ for the close II solution) are heavier than Earth, while the mass estimated from the wide solution $\left(M_{\text {planet }} \sim 0.6 M_{\oplus}\right)$ is below the mass of Earth.

Among the three 2L1S solutions, we exclude the wide solution for three major reasons. First, the model fit of the wide solution is worse than the close II solution by $\Delta \chi^{2}=19.2$ when the higher-order models are compared. This $\chi^{2}$ difference is already quite large and significantly disfavors the wide solution by itself. However, the wide solution yields the best fit when the standard models are compared, and thus this alone does not provide a crucial clue for disfavoring the wide solution. Second, only a very small fraction of Bayesian output is consistent with the flux constraint. According to the Bayesian analysis, the median values of the lens mass and distance are $\left(M_{\text {host }}, D_{\mathrm{L}}\right) \sim\left(1 M_{\odot}, 2.2 \mathrm{kpc}\right)$, and thus the lens would have a dereddened magnitude of $I_{0} \sim 15.8$. By adopting $A_{I} \sim 1.5$ considering that most of the dust is very nearby because it is a high latitude field $\left(b \sim 4^{\circ}\right)$, the apparent magnitude of the lens would be $I \sim 17.3$, which is substantially brighter than the blend with $I_{b} \sim 18.8$. Taking account of the fact that the lens mass could be more than $1 \sigma$ lower than the median, the lower limit of the lens mass is $M_{\min } \sim 0.7 M_{\odot}$, and the corresponding lower limit of the lens magnitude is $I_{\mathrm{L}, \min } \sim 19.0$. Considering that the flux from the lens comprises a small fraction of the blending flux as discussed in Sect. 4, the lens should be much fainter than $I_{b}$. This implies that only a very small fraction of Bayesian output is consistent with the constraint given by the blending flux. Finally, the wide solution results in an overall Bayesian probability that is much lower than the other solutions. The relative ratios of the overall Bayesian probabilities are $1.00: 0.83: 0.04$ for the close I, close II, and wide solutions, respectively. This implies that the probability of a lensing event with $M$ and $D_{\mathrm{L}}$ corresponding to the wide solution is much lower than the probability of an event with the physical lens parameters corresponding to the close solutions.

Despite the variation depending on the remaining solutions, the estimated mass indicates that KMT-2021-BLG-0912Lb is a super Earth, which is defined as a planet having a mass higher than that of Earth, but substantially lower than those of the Solar System's ice giants (Valencia et al. 2007). The number of super Earth planets detected by microlensing is rapidly increasing since the onset of high-cadence surveys. The microlensing planets with $M \lesssim 10 M_{\oplus}$ are listed in Table 3 of Han et al. (2021). With an additional planet KMT-2020-BLG-0414LAbB (Zang et al. 2021), that has been detected since the publication of their paper, there exist 19 super-Earth microlensing planets with $M \lesssim 10 M_{\oplus}$, including the one we report in this work.
Among these planets, 16 planets were detected during the last 6 years after the full operation of the KMTNet survey. This well demonstrates the importance of high-cadence global surveys in detecting low-mass planets.

\section{Conclusion}

We investigated the light curve of the lensing event KMT-2021BLG-0912, which exhibited a very short anomaly relative to a single-lens single-source form. Although this event reached a high magnification and had substantial followup data, the anomaly occurred $\sim 11.5$ days after the peak when the event was only magnified by $A \sim 6$ and no longer receiving followup observations. Hence, the discovery and characterization of this anomaly relied entirely on survey observations taken at a cadence of several points per night from multiple locations.

From the modeling of the light curve under various interpretations, we found four solutions, in which three solutions were found under the assumption that the lens was composed of two masses, and the other solution was found under the assumption that the source was comprised of a binary-star system. The 1L2S model was rejected based on the contradiction that the faint source companion was bigger than its primary, and one of the $2 \mathrm{~L} 1 \mathrm{~S}$ solutions was excluded from the combination of the relatively worse fit, blending constraint, and lower overall probability, leaving two surviving solutions with planet/host mass ratios of $\sim 2.8 \times 10^{-5}$ and $\sim 1.1 \times 10^{-5}$. A subtle central deviation might support the possibility of a tertiary lens component, either a binary companion to the host with a very large or small separation or a second planet lying at around the Einstein ring, but it was difficult to claim a secure detection due to the marginal fit improvement, lack of consistency among different data sets, and difficulty in uniquely specifying the nature of the tertiary component. With the observables of the event, it was estimated that the masses of the planet and host were $\sim\left(6.9 M_{\oplus}, 0.75 M_{\odot}\right)$ according to one solution and $\sim\left(2.8 M_{\oplus}, 0.80 M_{\odot}\right)$ according to the other solution, indicating that the planet was a super Earth around a K-type star, regardless of the solutions. The fact that 16 , including the one reported in this work, out of 19 microlensing super-Earth planets with $M \lesssim 10 M_{\oplus}$ were detected during the last 6 years well demonstrates the importance of high-cadence surveys in detecting very low-mass planets.

Acknowledgements. Work by C.H. was supported by the grants of National Research Foundation of Korea (2020R1A4A2002885 and 2019R1A2C2085965). S.D. and Z.L. acknowledges the science research grants from the China Manned Space Project with NO. CMS-CSST-2021-A11. W.Zang, S.M. and H.Y. acknowledge support by the National Science Foundation of China (Grant No. 11821303 and 11761131004). This research uses data obtained through the Telescope Access Program (TAP), which has been funded by the TAP member institutes. J.C.Y. acknowledges support from N.S.F Grant No. AST-2108414. This research has made use of the KMTNet system operated by the Korea Astronomy and Space Science Institute (KASI) and the data were obtained at three host sites of CTIO in Chile, SAAO in South Africa, and SSO in Australia. Work by I.K. was supported by JSPS KAKENHI Grant Number 20J20633. Work by D.P.B., A.B., and C.R. was supported by NASA through grant NASA-80NSSC18K027. T.S. acknowledges the financial support from the JSPS, JSPS23103002, JSPS24253004, and JSPS2624702. Work by N.K. is supported by JSPS KAKENHI Grant Number JP18J00897. The MOA project is supported by J SPS KAK-ENHI Grant Number JSPS24253004, JSPS26247023, JSPS23340064, JSPS15H00781, JP16H06287, 17H02871, and 19KK0082.

\section{References}

Alard, C. 2000, A\&AS, 144, 363

Alard, C., \& Lupton, R. H. 1998, ApJ, 503, 325

Albrow, M. 2017, MichaelDAlbrow/pyDIA: Initial Release on Github, Versionv1.0.0, Zenodo, doi:10.5281/zenodo.268049 
Albrow, M., Horne, K., Bramich, D. M., et al. 2009, MNRAS, 397, 2099 Batista, V., Gould, A., Dieters, S., et al. 2011, A\&A, 529, 102 Beaulieu, J.-P., Bennett, D. P., Fouqué, P, et al. 2006, Nature, 439, 437 Bensby, T., Yee, J. C., Feltzing, S., et al. 2013, A\&A, 549, A147 Bond, I. A., Abe, F., Dodd, R. J., et al. 2001, MNRAS, 327, 868 Bessell, M. S., \& Brett, J. M. 1988, PASP, 100, 1134

Bozza, V. 1999, A\&A, 348, 311

Chung, S.-J., Han, C., Park, B.-G., et al. 2005, ApJ, 630, 535

Dominik, M. 1998, A\&A, 329, 361

Dominik, M. 1999, A\&A, 349, 108

Dong, S., Gould, A., Udalski, A., et al. 2009, ApJ, 695, 970

Gaudi, B. S. 1998, ApJ, 506, 533

Gaudi, B. S., \& Han, C. 2004, ApJ, 611, 528

Gaudi, B. S., Naber, R. M., \& Sackett, P. D. 1998, ApJ, 502, L33

Gould, A. 1992, ApJ, 392, 442

Gould, A. 2000, ApJ, 542, 785

Gould, A., \& Loeb, A. 1992, ApJ, 396, 104

Griest, K., \& Safizadeh, N. 1998, ApJ, 500, 37

Griest, K., \& Hu, W. 1992, ApJ, 397, 362

Han, C. 2006, ApJ, 638, 1080

Han, C., Chang, H.-Y., An, J. H., \& Chang, K. 2001, MNRAS, 328, 986

Han, C., \& Gould, A. 1995, ApJ, 447, 53

Han, C., \& Gould, A. 1997, ApJ, 480, 196

Han, C., \& Gould, A. 2003, ApJ, 592, 172

Han, C., Gould, A., Albrow, M., et al. 2021, A\&A, submitted

Jung, Y. K., Han, C., Udalski, A., et al. 2021, AJ, 161, 293

Jung, Y. K., Udalski, A., Gould, A., et al. 2018, AJ, 155, 219

Kervella, P., Thévenin, F., Di Folco, E., \& Ségransan, D. 2004, A\&A, 426, 29

Kim, S.-L., Lee, C.-U., Park, B.-G., et al. 2016, JKAS, 49, 37

Nataf, D. M., Gould, A., Fouqué, P., et al. 2013, ApJ, 769, 88

Robin, A. C., Reylé, C., Derriére, S., \& Picaud, S. 2003, A\&A, 409, 523

Ryu, Y.-H., Hwang, K.-H., Gould, A., et al. 2021, AJ, 162, 96

Schechter, P. L., Mateo, M., \& Saha, A. 1993, PASP, 105, 1342

Skowron, J., Udalski, A., Gould, A., et al. 2011, ApJ, 738, 87

Tomaney, A. B., \& Crotts, A. P. S. 1996, AJ, 112, 2872

Udalski, A., Jaroszyński, M., Paczyński, B., et al. 2005, ApJ, 628, L109

Udalski, A., Szymański, M. K., \& Szymański, G. 2015, Acta Astron., 65, 1

Valencia, D.,:10440 Sasselov, D. D., \& O’Connell, R. J. 2007, ApJ, 656, 545

Yee, J. C., Shvartzvald, Y., Gal-Yam, A., et al. 2012, ApJ, 755, 102

Yoo, J., DePoy, D. L., Gal-Yam, A., et al. 2004, ApJ, 603, 139

Zang, W.-C., Han, C., Kondo, I. et al. 2021, Res. Ast. Astrophys., 9, 239

Zang, W., Penny, M. T., Zhu, W., et al. 2018, PASP, 130:10440

Zhu, W., Penny, M., Mao, S., et al. 2014, ApJ, 788, 73
1 Department of Physics, Chungbuk National University, Cheongju 28644, Republic of Korea

e-mail: cheongho@astroph.chungbuk.ac.kr

2 Institute of Natural and Mathematical Sciences, Massey University, Auckland 0745, New Zealand

${ }^{3}$ Center for Astrophysics | Harvard \& Smithsonian 60 Garden St., Cambridge, MA 02138, USA

${ }^{4}$ Department of Astronomy, Tsinghua University, Beijing 100084, China

5 University of Canterbury, Department of Physics and Astronomy, Private Bag 4800, Christchurch 8020, New Zealand

${ }^{6}$ Korea Astronomy and Space Science Institute, Daejon 34055, Republic of Korea

7 Max Planck Institute for Astronomy, Königstuhl 17, D-69117 Heidelberg, Germany

8 Department of Astronomy, The Ohio State University, 140 W. 18th Ave., Columbus, $\mathrm{OH} 43210$, USA

9 Department of Particle Physics and Astrophysics, Weizmann Institute of Science, Rehovot 76100, Israel

${ }^{10}$ School of Space Research, Kyung Hee University, Yongin, Kyeonggi 17104, Republic of Korea

11 Korea University of Science and Technology, 217 Gajeong-ro, Yuseong-gu, Daejeon, 34113, Republic of Korea

12 Institute for Space-Earth Environmental Research, Nagoya University, Nagoya 464-8601, Japan

13 Code 667, NASA Goddard Space Flight Center, Greenbelt, MD20771, USA

14 Department of Astronomy, University of Maryland, College Park, MD 2074

15 Department of Earth and Space Science, Graduate School of Science, Osaka University, Toyonaka, Osaka 560-0043, Japan

16 Department of Earth and Planetary Science, Graduate School of Science, The University of Tokyo, 7-3-1 Hongo, Bunkyo-ku, Tokyo 113-0033, Japan

17 Instituto de Astrofśica de Canarias, Vía Láctea s/n, E-38205 La Laguna, Tenerife, Spain

18 Department of Astronomy, Graduate School of Science, The University of Tokyo, 7-3-1 Hongo, Bunkyo-ku, Tokyo 113-0033, Japan

19 National Astronomical Observatory of Japan, 2-21-1 Osawa, Mitaka, Tokyo 181-8588, Japan

20 Department of Physics, University of Auckland, Private Bag 92019, Auckland, New Zealand

21 Institute of Space and Astronautical Science, Japan Aerospace Exploration Agency, 3-1-1 Yoshinodai, Chuo, Sagamihara, Kanagawa, 252-5210, Japan

22 Department of Physics, The Catholic University of America, Washington, DC 20064, USA

23 University of Canterbury Mt. John Observatory, P.O. Box 56, Lake Tekapo 8770, New Zealand

24 Kumeu Observatory, Kumeu, New Zealand

25 Kavli Institute for Astronomy and Astrophysics, Peking University, Yi He Yuan Road 5, Hai Dian District, Beijing 100871, China

26 Possum Observatory, Patutahi, New Zealand

27 Centre for Astrophysics, University of Southern Queensland, Toowoomba, Queensland 4350, Australia

28 Department of Astronomy, School of Physics, Peking University, Yi He Yuan Road 5, Hai Dian District, Beijing 100871, China

29 National Astronomical Observatories, Chinese Academy of Sciences, Beijing 100101, China

30 School of Physics and Astronomy, Tel-Aviv University, Tel-Aviv 6997801, Israel

31 Department of Physics and Astronomy, Louisiana State University, Baton Rouge, LA 70803 USA 